\title{
The Bionic Research on Avian Visual Structure in Multi-Target Monitoring
}

\author{
Bingxin Wang, Binquan Zhang, Sun'an Wang ${ }^{*}$ and Dehong Yu \\ School of Mechanical Engineering, Xi'an Jiaotong University, Xi'an 710049, Shaanxi, China \\ sawang@mail.xjtu.edu.cn
}

\begin{abstract}
Keywords: predator-prey relation, dual fovea, bionic design, visual monitoring, multi target Abstract. The key techniques of visual monitoring contain searching and measuring multiple targets in large region. However, many intelligent monitoring systems today cannot satisfy these requirements simultaneously. By analyzing the features of creatural vision in predator-prey relation, the relationships among visual characteristic parameters were obtained. On this basis, a multi-target visual monitoring system with avian bifoveate feature was designed with the idea and technology of bionics in this paper. This system is characterized by wide vision field in prey and developed binocular vision in predators, and also expands animal visual performance. It has the ability to synchronously achieve the goals of rapidly searching and precisely positioning multiple objects in all directions. This research also provides new insight into bionics design of visual monitoring systems.
\end{abstract}

\section{Introduction}

The function of fitting the living conditions, which provides assistance to engineering and technical difficulties, is preserved by biological evolution and natural selection over billions of years. As an important source for most vertebrates to get information from the surrounding environment, animal vision systems have been widely studied in the field of bionics. Asfour [1] used wide-angle lens as the human retina and narrow-angle lens as central fovea to implement humanoid visuo-motor behaviors. Jia [2] utilized a pair of fisheye lens to mimic the horse's wide monocular vision and narrow binocular vision, which achieved the detection of obstacles and surroundings. By simulating the visual behavior of chameleon in the feeding process, Zhao [3] and Wan [4] respectively borrowed the features from chameleon's independent and synergic eye movement to get better monitoring view. Gao [5] adopted saccade mode and binocular mode to recognize and track pedestrians through imitating eagle's bifoveate structure.

Because of the impact of structure, most bionic vision systems cannot synchronously satisfy the three goals of searching widely, positioning precisely and tracking multi-target. In other words, if a target has been locked, it will be difficult to find a newly emerging one.

Focusing on these problems, a bionic visual monitoring system was designed though combining the visual features of predator-prey relation and employing avian bifoveate structure. This system has the capacity to widely search, accurately locate and measure distance to multiple appetizing target at the same time. Thus, it solves the compatibility issue of the above goals. Firstly, the visual features among predators, prey and in-between species were analyzed in this paper. And the variation rules of parameters were obtained, according to the visual characteristic parameters in birds as prototype. Then, avian bifoveate feature was introduced as bionic functional and structural archetype. Next, the bionic visual monitoring system with dual fovea was designed. Finally, the operating principle and semi-system experiment results were shown.

\section{The analysis of animal vision system}

As the Chinese saying goes, the mantis stalks the cicada, unaware of the oriole behind. Therefore, mantis should protect itself from oriole behind by searching timely, when playing the role as predator. For preying and stymieing any attack, animals at the different positions in food chain have evolved corresponding vision systems. 
The creatural visual characteristic parameters generally include binocular overlap (BO), lateral vision (LV), monocular vision field (MVF), vision field (VF) and the direction of the optic axes of the eye relative to the midline of the head (DOA), which are shown in Fig. 1 and are usually specified in degree. The relationship among the parameters is:

$$
\mathrm{VF}=\mathrm{BO}+\mathrm{LV}_{\text {left }}+\mathrm{LV}_{\text {right }}
$$

$$
\mathrm{LV}=\mathrm{MVF}-\mathrm{BO}
$$

According to the research of predator and prey vision systems by Jones [7], Waldvogel [8] and Pettigrew [9]:

- In order to capture preies accurately, predators such as falcon, eagle, leopard and tiger (Fig. 2) have evolved frontally placed eyes. This structure has wide bioncular overlap and narrow vision field.

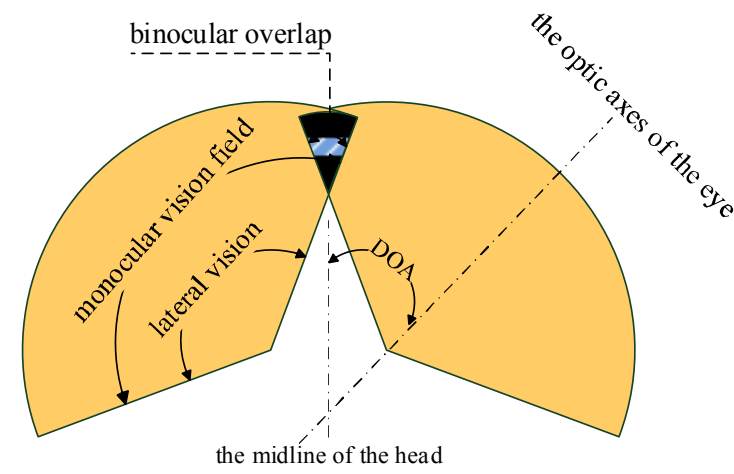

Fig. 1 Characteristic parameters of creatural vision system

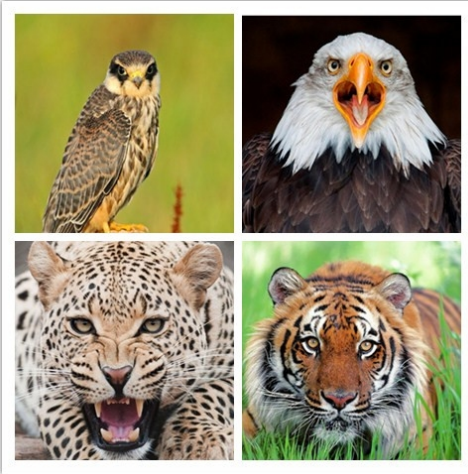

Fig. 2 Typical predators

- To find the potential attackers in any direction as swiftly as possilbly, prey such as pigeon, horse, rabbit and gold fish (Fig. 3) have evolved laterally placed eyes. This structure gives them almost $360^{\circ}$ omnidirectional vision field and narrow binocular overlap.

- The in-between species such as titmouse, tawny frogmouth, lizard and frog (Fig. 4) not only need hunt their preies, but also have to avoid attacks from other large animals. Hence, they change the range of vision field and binocular overlap thought wide eye movement, which can ensure them adpot to different roles in predator-prey relation. For instance, when hunting, they will gather their eyes to increase binocular overlap; when being hunted, they will separate their eyes to enlarge vision field.

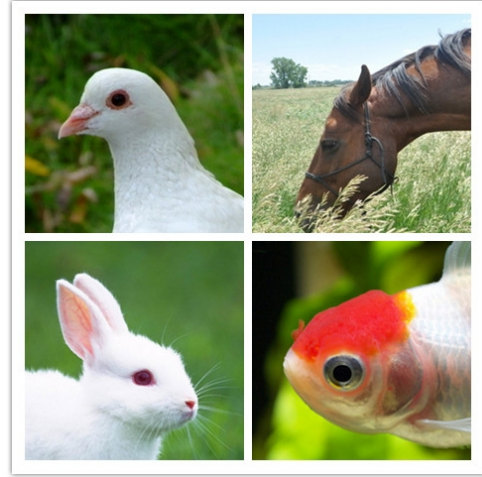

Fig. 3 Typical prey

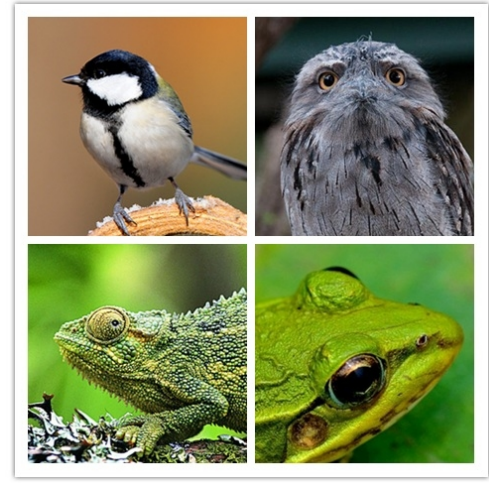

Fig. 4 In-between species

On the basis of above qualitative analysis, we performed quantitive analysis of conversion trends on avian visual characteristic paramenters (Table 1), and took typical highly predatory birds, wing-feeding insectivorous birds and prey birds for example. As shown in Table 1, pigeon as typical prey has the narrowest binocular overlap, the widest vision field and the widest DOA. Barn owl as typical predator has the widest binocular overlap and the narrowest vision field. Other birds' data are not beyond these parameters. 
Table 1 The visual characteristc parameters of birds $[6,7,10]$

\begin{tabular}{|c|c|c|c|c|}
\hline name & $\mathrm{DOA} /{ }^{\circ}$ & $\mathrm{BO} /{ }^{\circ}$ & $\mathrm{MVF} /{ }^{\circ}$ & $\mathrm{VF} /{ }^{\circ}$ \\
\hline pigeon & 72 & 20 & 160 & 300 \\
\hline little eagle & 33 & 20 & & \\
\hline cooper's hawk & $66.93 \pm 1.29$ & 36 & 168 & 300 \\
\hline American kestrel & $63.83 \pm 1.47$ & 33 & 163 & 293 \\
\hline Red tailed hawk & $68.97 \pm 1.25$ & 33 & 155.5 & 278 \\
\hline tawny owl & 28 & 48 & & \\
\hline owl & 27 & 48 & & \\
\hline titmouse & & $53 / 78$ & & \\
\hline barn owl & & $60-70$ & $105-110$ & 150 \\
\hline
\end{tabular}

Using these data above, the authors of this paper did curve fitting of every two parameters (Fig. 5). The results show the positive correlation between monocular vision field and vision field. What's more, the correlation is almost a linear relationship:

$$
\mathrm{VF}=3 \cdot \mathrm{MVF}-180^{\circ}
$$

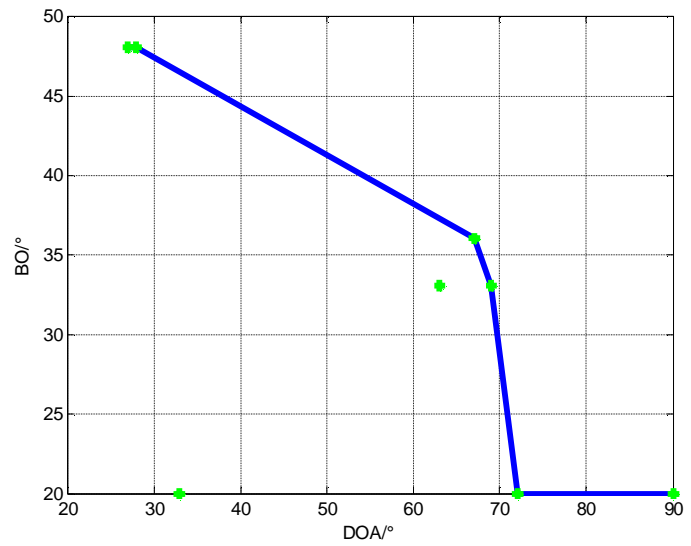

(a) The relation between $\mathrm{BO}$ and DOA

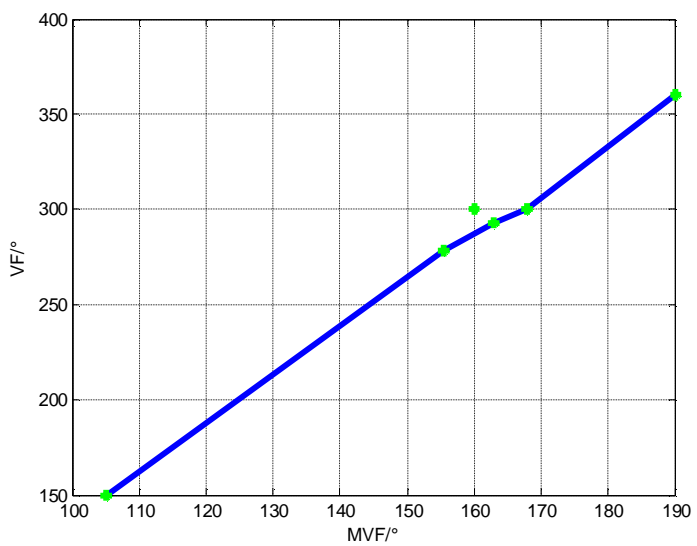

(b) The relation between VF and MVF

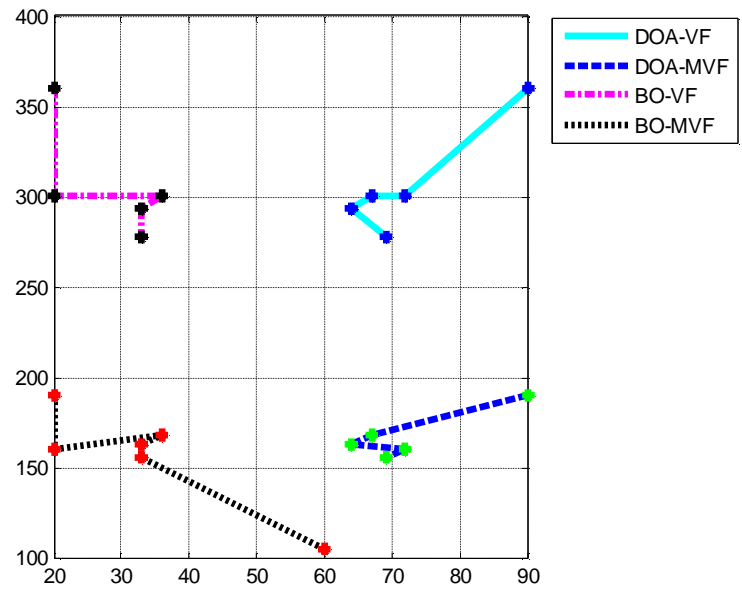

(c) The relations among other parameters ${ }^{\circ}$

Fig. 5 The relationships among avian visual characterstic parameters

It also demonstrates negative correlation between DOA and binocular overlap. There are not clear functional relationships among other parameters, but the overall trend is: With predatory increasing, DOA, monucular vision field and vision field show decreasing trend, while binocular overlap shows increasing trend. This has a consistent conclusion with the visual characteristic theoretical analysis. So 
our bionic purpose is to combine visual features of prey and predators to make manmade system have panoramic view and wide binocular overlap.

\section{The bifoveate structure of birds}

In the predator-prey relation, animals at different positions in food chain have evolved different dominant visual structures to adapt to the environment. Birds not only need precisely shuttle in trees and catch insets, but also avoid the capture of other predators [8]. As a result, avian vision gradually has the in-between species visual characteristics, that is, they can both search in wide range and position accurately. Nonetheless, they don't depend on the strong eye movement to converse these two capacities. Birds have evolved a special eye structure - dual fovea, and unite it with head movement to observe the surroundings.

Bifoveate structure has been found in raptors (eagle, falcon, etc.), other wing-feeding insectivorous birds (swallow, swift, etc.) and Anolis lizards [7, 11, 12]. Dual fovea include a high-acuity deep central fovea and a shallow temporal fovea used to form binocular vision (Fig. 6). In other birds (as pigeon), they only have a real fovea, but they have red area, which has the same position and function as temporal fovea [13].

Tucker [11] also found that which fovea to use is related to the logarithmic spiral path in predation process (Fig. 7). At a distance closer than $8 \mathrm{~m}$, raptors spent more time looking at the object straight using temporal fovea (shallow fovea). Then it will be easier for them to aim and track the target precisely. But as the distance over than $21 \mathrm{~m}$, raptors spent more time looking at their sideways using central fovea (deep fovea) [7, 11].

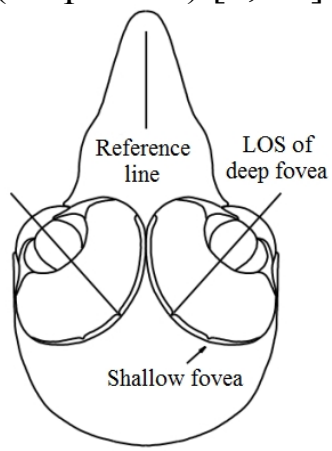

Fig. 6 The sketch of avian dual fovea [11]

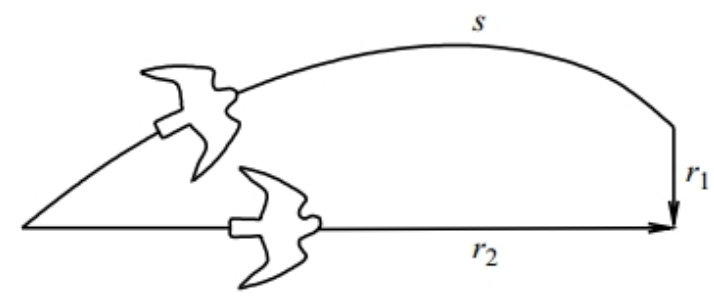

Fig. 7 The way of raptor catching preies [11]

\section{The design of bionic visual monitoring system}

In actual monitoring applications, visual monitoring systems need find, recognize and track targets autonomously. The usual way is that when a specific target has been found by widely searching, the system will lock and track it. However, these systems only imitate a single predator or prey visual feature. If applied to multi-target recognition and tracking occasion, the system will be difficult to find and recognize other emerging target quickly.

To simultaneously achieve the goals of rapidly searching and precisely positioning multiple targets in all directions, we combined the visual features of predators and prey and adopted avian bifoveate structure to design a new visual monitoring system. In overall design (Fig. 8), two fisheye lens with $180^{\circ} \sim 220^{\circ}$ visual angle are used to form $360^{\circ}$ omnidirectional vision field by back-to-back mounting. Four normal lens with $30^{\circ} \sim 60^{\circ}$ visual angle are used to simulate dual fovea for tracking targets. The normal lens are installed symmetrically. By rotating the two closest normal lens till parallel, there will be at least $30^{\circ}$ binocular overlap. The whole system also can rotate to imitate birds' head movement.

As a consequence, the system in this paper has the visual features shown Table 1 of predators and prey concurrently. This guarantees omnidirectional vision field and a wide range of binocular overlap, and also meets the application requirements of widely searching and precisely positioning multiple target at the same time. 


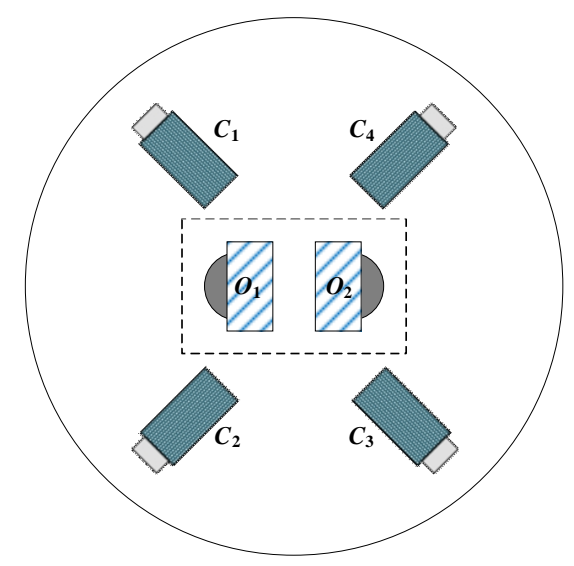

(a) Top view

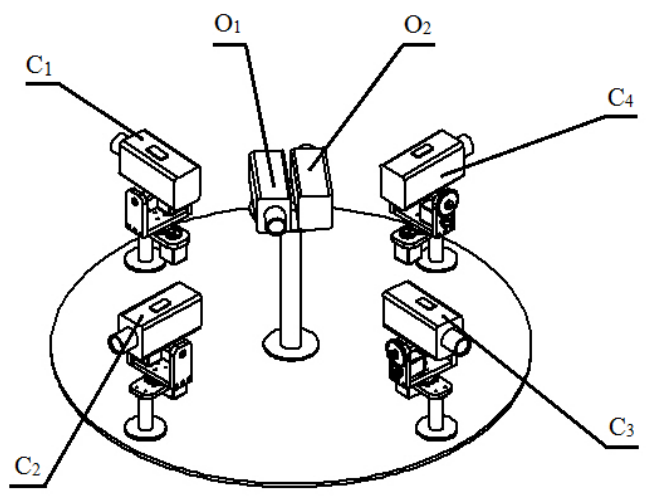

(b) Dimentional view

Fig. 8 The structure of the bionic visual monitoring system based on dual fovea

\section{The working principle of bionic multi-target visual monitoring system}

The working principle of this system is shown in Fig. 9. The system is divided into two basic working modes: searching mode and tracking mode. Tracking mode also has two working submode: staring submode and measuring submode.
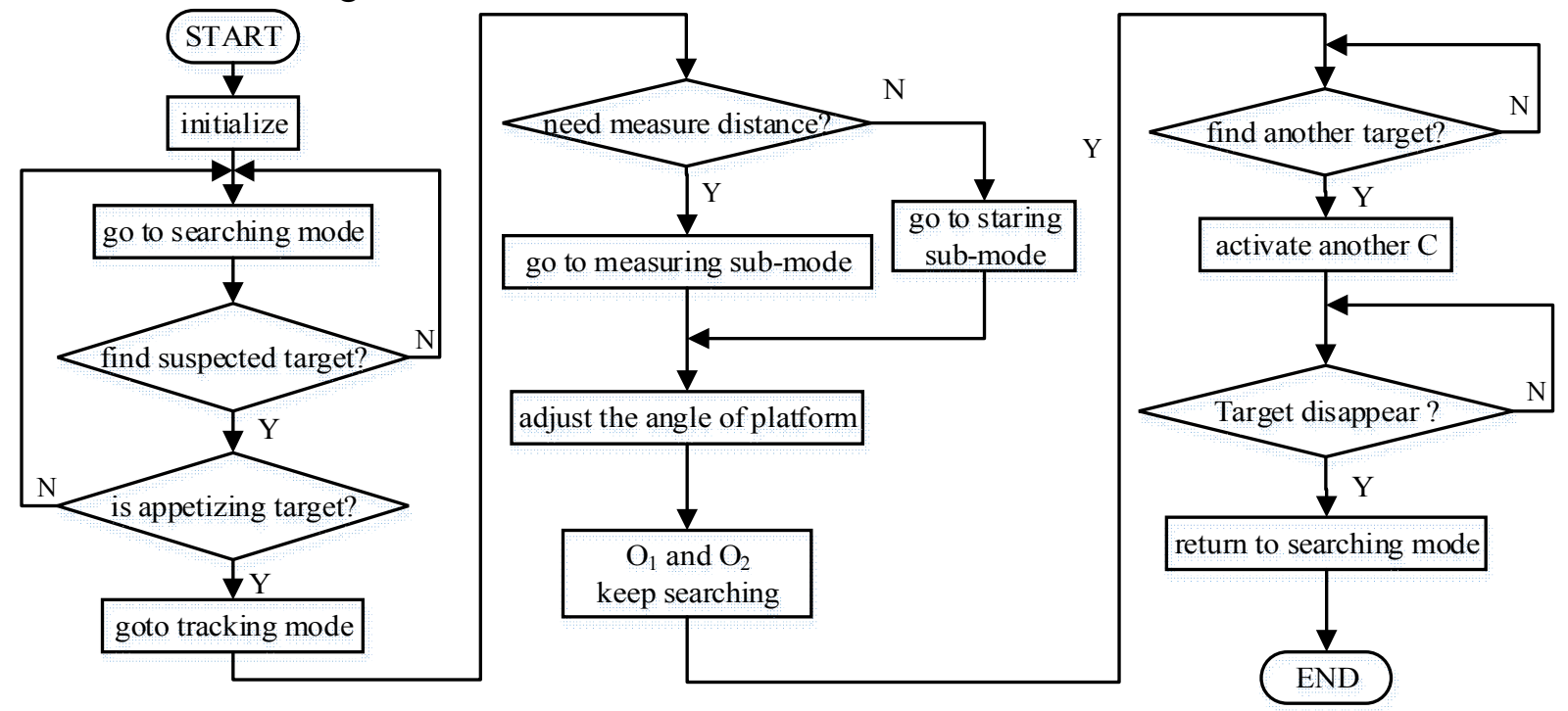

Fig. 9 The main working process of bionic multi-target visual monitoring system

If the system doesn't find any target, it will work in searching mode, and only $\mathrm{O}_{1}$ and $\mathrm{O}_{2}$ are activated. When suspected target is found, the system will judge whether the target is the real appetizing one. If the target is, the system will change to tracking mode.

For observing target in tracking mode, system will switch to staring submode, and the closest normal lens $\mathrm{C}_{\mathrm{i}}(\mathrm{i}=1,2,3,4)$ will be activated as central fovea. For measuring distance, system will switch to measuring submode, and the closest two normal lens $C_{i}$ and $C_{j}(i, j=1,2,3,4)$ will be activated as temporal fovea and rotated to the target. In the process, the system also can adjust the angle between lens and targets to catch object better, by rotating the whole platform.

$\mathrm{O}_{1}$ and $\mathrm{O}_{2}$ are invariably activated for searching and verifying other suspected target. When new target appears, the system can activate other normal lens and coordinate their work submodes to gaze or measure the new and original targets together.

\section{Experiments}

In the indoor environment, we did the experiments of staring and measuring multi-target using semi-system without rotating the whole platform. 
The semi-system includes one $150^{\circ}$ digital camera $\mathrm{O}_{1}$ (S-YUE WX-152) and two $40^{\circ}$ digital cameras $\mathrm{C}_{1}$ and $\mathrm{C}_{2}$ (AONI D881HD720P). The appetizing targets' sizes are $10 \mathrm{~cm} \times 15 \mathrm{~cm}$ and $13 \mathrm{~cm} \times 14 \mathrm{~cm}$, and their distance to experimental platform is more than $3 \mathrm{~m}$.

Experiment 1 is about single target staring. $\mathrm{O}_{1}$ was used to imitate MVF of prey. $\mathrm{C}_{1}$ and $\mathrm{C}_{2}$ were used to imitate dual fovea. Taking mimicing left eye for example, when target appeared in posterior area (Fig. 10(a)(b)), $\mathrm{C}_{1}$ was activated and rotated to observe target as central fovea (Fig. 10(c)). $\mathrm{C}_{2}$ remained in beginning position at this time(Fig.10(d)). When the target moved to anterior area, $\mathrm{C}_{1}$ was reverted to its original position, and $\mathrm{C}_{2}$ was rotated to observe target. This experiment shows that the semi-system can keep target being captured by $\mathrm{C}_{1}$ or $\mathrm{C}_{2}$ in the range of $180^{\circ}$.

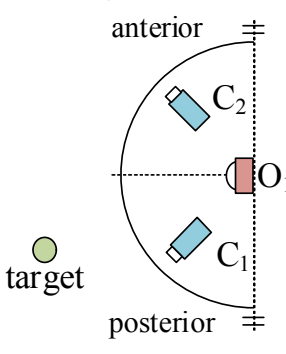

(a) exp. design

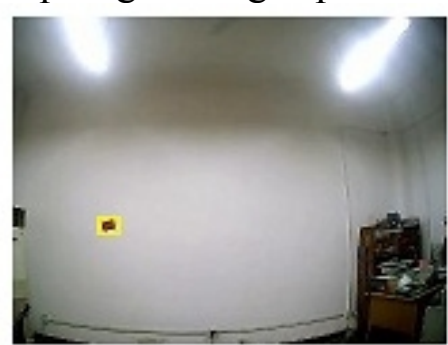

(b) image of $\mathrm{O}_{1}$

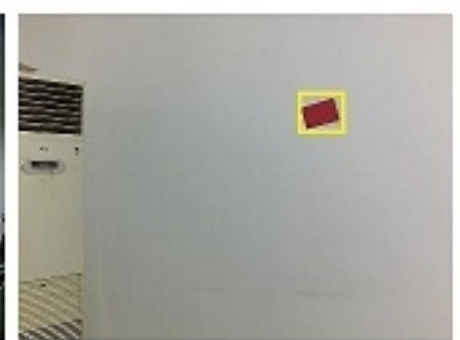

(c) image of $\mathrm{C}_{1}$

Fig. 10 Exp. 1: staring a target using left eye

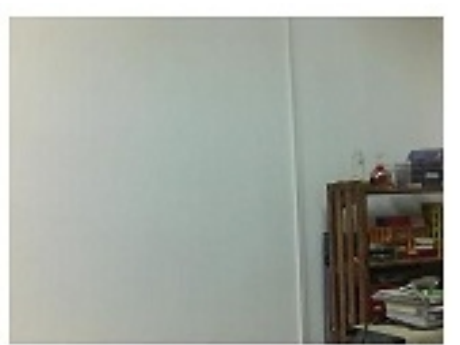

(d) image of $\mathrm{C}_{2}$

Experiment 2 is about measuring distance to single target. $\mathrm{O}_{1}$ was utilized to imitate bird's frontal vision view of head. $\mathrm{C}_{1}$ and $\mathrm{C}_{2}$ were utilized to mimic temporal fovea in left eye and right eye. When target appeared in the middle of image (Fig. 11(a)(b)), $\mathrm{C}_{1}$ and $\mathrm{C}_{2}$ were activated and rotated toward target to make sure that the object could be found in both images (Fig. 11(c)(d)). This experiment demonstrates that the semi-system can accomplish distance measurement using stereo vision. In addition, incorporating exp. 1 and exp. 2, the system can perfectly replicate how raptors use dual fovea in predation process.

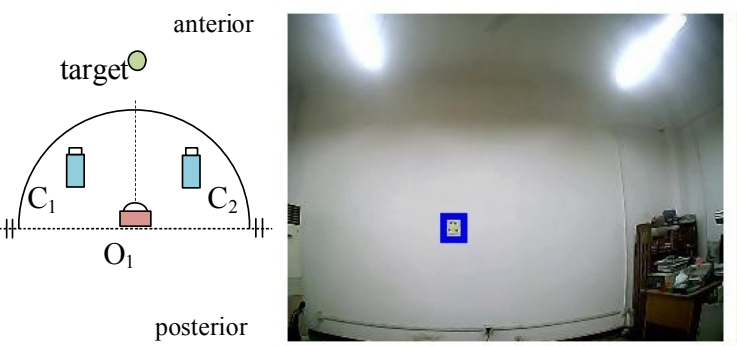

(a) exp. design

(b) image of $\mathrm{O}_{1}$

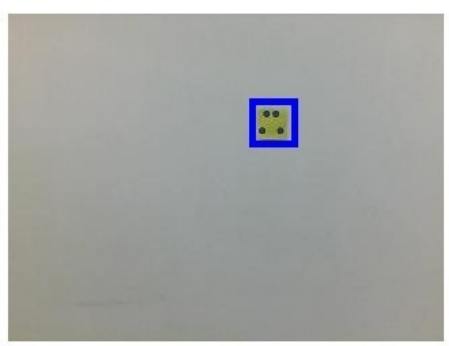

(c) image of $\mathrm{C}_{1}$

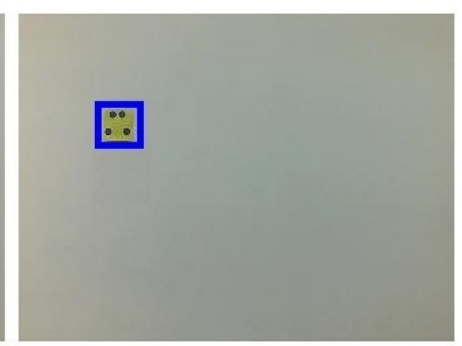

(d) image of $\mathrm{C}_{2}$

Fig. 11 Exp.2: measuring distance to a target in anterior area

Experiment 3 expands animal visual performance by using dual fovea to staring two targets at the same time (Fig. 12(a)(b)). $\mathrm{O}_{1}$ was used to form monocular vision field of prey. $\mathrm{C}_{1}$ and $\mathrm{C}_{2}$ were used to form two different fixation points. According to the positions of targets, system utilized $\mathrm{C}_{1}$ and $\mathrm{C}_{2}$ to observe targets separately (Fig. 12(c)(d)).

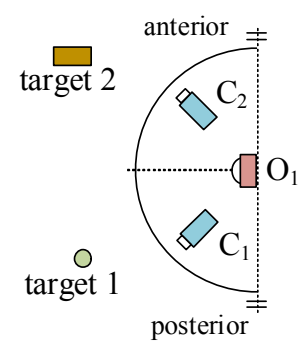

(a) exp. design

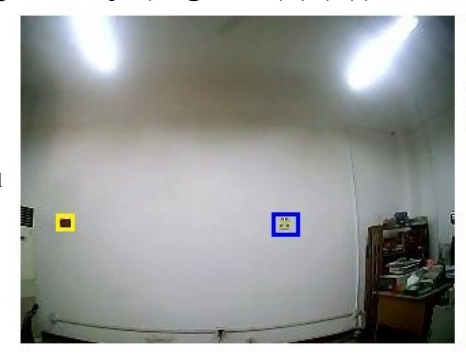

(b) image of $\mathrm{O}_{1}$

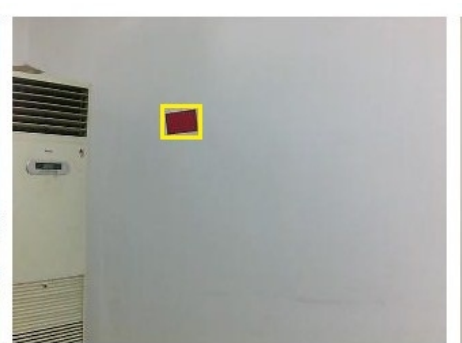

(c) image of $\mathrm{C}_{1}$

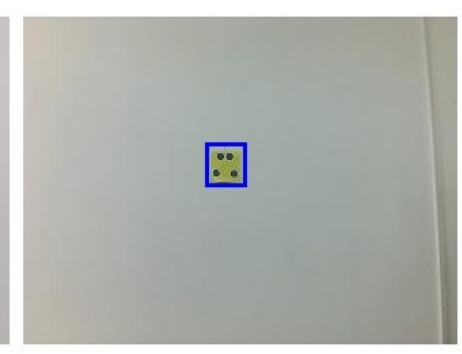

(d) image of $\mathrm{C}_{2}$

Fig. 12 Exp. 3: staring two targets simultaneously

All these experiments show that: wide-angle lens can achieve target searching in almost $180^{\circ}$ vision angle without rotating platform. Normal lens can accompish target locking and tracking in almost $180^{\circ}$ 
vision angle by changing their working mode, which also cut down the noise for target recognition in surrounding environment.

\section{Conclusion}

We combined the visual features of predators and prey, and utilized dual fovea visual structure to design a bionic visual monitoring system. This system not only imitates avian vision, but also transcends birds' own capacities. It provides a new bionic idea to solve the compatibility among wide searching, accurately positioning and multi-target tracking in engineering. We will build the complete system and carry on further research of multi-target recognition and tracking by combining head movement and eye movement to bionic control strategy.

\section{Acknowledgement}

The work presented in this paper is supported by National Natural Science Foundation of China (No. 51375368).

\section{References}

[1] T Asfour, K Welke, P Azad, et al. The karlsruhe humanoid head, J. in: Humanoid Robots, 2008. Humanoid 2008. 8th IEEE-RAS International Conference on IEEE, 1-3 2008 (2008) 447-453.

[2] H Jia, S Li. Scene analysis based on horse vision system, C. MVA (2011) 267-270.

[3] L Zhao, L Kong, Y Wang. Error analysis of binocular active hand-eye visual system on parallel mechanisms, C. Information and Automation, 2008. ICIA 2008. International Conference on IEEE, (2008) 95-100.

[4] D Wan. Research on Dual-PTZ-Camera system, D. Tsinghua University. (2012)

[5] Y Gao, X. Zhang. Active zoom cameras control system using stereo parallax, C. Secunity Technology(ICCST), 2010 IEEE International Camahan Conference on IEEE, (2010) 418-424.

[6] CT O'Rourke, MI Hall, T Pitlik, et al. Hawk eyes I: diurnal raptor differ in visual fields and degree of eye movement, J. Plos one. 5.9 (2010) e12802.

[7] MP Jones, KE Pierce Jr, D ward. Avian vision: a review of form and function with special consideration to birds of prey, J. Journal of Exotic Pet Medicine. 16.2 (2007) 69-87.

[8] JA Waldvogel. The birds's eye view, J. American Scintist. 78 (1990) 342-353.

[9] JD Pettigrew. Evolution of binocular vision, J. Visual neuroscience. (1986) 208-222.

[10] MF Land. Eye movement of vertebrates and their relation to eye form and function, J. Journal of Comparative Physiology A. 201 (2015) 195-214.

[11] VA Tucker. The deep fovea, sideways vision and spiral flight paths in raptors, J. Journal of Experimental Biology, 203.24 (2000) 3745-3754.

[12] KV Fite, BC Lister. Bifoveal vision in Anolis lizards, J. Brain, Behavior \& Evolution. 19.3-4 (1981) 144-43.

[13] PG Clarke, D Whitteridge. The projection of the retina, including the 'red area' on to the optic tectum of the pigeon, J. Quarterly Journal of Experimental Physiology \& Cognate Medical Sciences. 61.4 (1976) 351-358. 